\title{
What's new for the clinician? Summaries of and excerpts from recently published papers
}

\section{Can hyaluronan injections augment deficient papillae at implant-supported crowns in the anterior maxilla?}

BertI K, Gotfredsen K, Jensen SS, Bruckmann C, Stavropoulos A. Clinical Oral Implants Research 2017; 28: 1054-61.

The presence or absence of the interproximal papilla is of great concern to periodontists, restorative dentists, and to the patients. The loss of papilla can lead to cosmetic deformities (so-called "black triangle disease"), phonetic problems (space allows passage for the air or saliva), and lateral food impaction. Open gingival embrasures, also called "black triangles," are often the result of attachment loss in the interproximal area, or may be due to surgical trauma, and are rather common at implant-supported crowns. ${ }^{1}$ Micro-surgical techniques have improved the aesthetic outcomes of periodontal/mucogingival interventions. However, the reconstruction of a missing papilla to close an open gingival embrasure remains among the most challenging and unpredictable surgical scenarios, due to anatomical and access restrictions. ${ }^{1}$ Thus, other treatment options are often proposed to close an open gingival embrasure, for example, orthodontics or restorative; however, these are rather time-consuming and/ or costly procedures. ${ }^{1}$ Promising results were described in terms of deficient papilla augmentation in recent reports including a few patient cases, with hyaluronan $(\mathrm{HY})$ application as injectable filler for reconstruction of interproximal papillae at implant-supported crowns and between teeth. ${ }^{1}$

$\mathrm{HY}$ is an important component of the extracellular matrix and is responsible for tissue resilience and volume due to its high hygroscopicity. ${ }^{1}$ Products containing HY are widely used in cosmetic medicine as facial fillers, for example, to reduce wrinkles or restore lost skin volume that often occurs with age due to - among other reasons -a decrease in the HY content of the skin. ${ }^{1}$ In dentistry, $\mathrm{HY}$ is used primarily due to its bacteriostatic, fungistatic, anti-inflammatory, anti-edematous, osteoinductive and pro-angiogenetic properties. In this context, a minimally invasive, simple, relatively inexpensive non-surgical technique for reconstruction of interdental papillae by $\mathrm{HY}$ injection appears as an attractive treatment approach. Bertl and colleagues (2017) reported on a randomized controlled clinical trial, with six months follow-up, that

\section{ACRONYM}

HY: hyaluronan

KT: $\quad$ keratinised tissue

MPIS: modified papilla index score

CP: contact point

BOP: bleeding on probing

sought to assess the effect of local HY injections to augment deficient interproximal papillae at implantsupported crowns in the anterior maxilla.

\section{MATERIALS AND METHODS}

The dental records of implant patients were screened for eligibility based on the following inclusion criteria: (i) $\geq 18$ years of age, (ii) implant restoration finalized >six months ago and (iii) at least one deficient papilla in the anterior maxilla (first premolar to first premolar) between a natural tooth and an implant-supported crown. The following clinical exclusion criteria were applied: (i) no contact point, (ii) inadequate plaque control (i.e., full-mouth plaque score $>20 \%$ ), (iii) probing depth (PD) $>5 \mathrm{~mm}$, or buccal gingival recession (GR) $>3 \mathrm{~mm}$, or $<2 \mathrm{~mm}$ keratinized tissue (KT) at the adjacent tooth/implant and (iv) systemic disorders (e.g., uncontrolled diabetes, malignancy) or regular intake of medications (e.g., steroids), affecting connective tissue metabolism. Oral and written informed consent was obtained from all participants prior to any intervention.

The test substance consisted of a commercially available $\mathrm{HY}$ product (Hyadent Barrier Gel); one $\mathrm{ml}$ of the gel contains $16 \mathrm{mg}$ cross-linked Na-hyaluronate and $2 \mathrm{mg} \mathrm{Na}$ hyaluronate. The control substance (placebo) consisted of physiological saline solution.

Patients who were included were allocated to either the test or control group according to a pre-defined randomization list and if the patient had two deficient papillae, either at the other site of the same implant-supported crown or at different implant-supported crowns in the anterior maxilla, both were allocated to the same treatment group (i.e., test or control); however, only one was chosen to be included in the analysis, by coin toss at baseline. Test and control substances were contained in identical and masked syringes to assure that patients were blinded to the treatment. However, due to the different consistency 
of the test and control substance (i.e., gel vs. fluid, respectively), the operator giving the injections could not be considered as blinded.

After administration of a local anesthesia gel ,the injection of the test or control substance was performed using a pressure syringe for standardized dose delivery $(0.06 \mathrm{ml}$ per "click") with a 30-gauge needle and a three-step technique was applied as per the manufacturer's recommendation: (i) creation of a reservoir in the mucosa immediately above the mucogingival junction (total amount- $0.18 \mathrm{ml}$ ), (ii) injection into the attached gingiva/mucosa just below the base of the deficient papilla (total amount - $0.12 \mathrm{ml}$ ), and (iii) injection 2-3 $\mathrm{mm}$ apically to the tip of the deficient papilla (total amount - $0.06 \mathrm{ml}$ ). The whole injection session was repeated once four weeks later.

Any discomfort during and within the first week after injection was recorded using a visual analogue scale (VAS) ranging from 0 (no pain) to 100 (worst pain).

Evaluation appointments were made before injection (baseline) and three and six months after the second injection. All parameters were assessed by a single blinded examiner, who was unaware of treatment group allocation, and the clinical measurements were recorded with a periodontal probe and rounded to the nearest half $\mathrm{mm}$.

The following parameters were assessed at baseline:

- Width of keratinized tissue (KT): distance (in $\mathrm{mm}$ ) from the gingival margin to the mucogingival junction at the mid-facial aspect of the adjacent tooth/implant.

- Gingival phenotype: a periodontal probe was placed in the sulcus at the mid-facial aspect of the adjacent tooth/implant, and if the periodontal probe was visible through the gingiva, the gingival phenotype was judged as "thin," or otherwise as "thick."

- Tissue texture and colour: gingival/mucosal tissue texture and colour of interproximal tissue (i.e., of the papilla) until the mid-facial aspect of the adjacent tooth/implant were compared to the surrounding tissues and classified as "more," "less," or "equally firm" and "more," "less," or "equally red," respectively

- The following parameters were assessed at baseline and after three and six months:

- Modified papilla index score (MPIS) Score 0 - no papilla is present; score 1 - less than half of the height of the papilla is present; score 2 - half or more of the height of the papilla is present, but not all the way to the contact point of the teeth; score 3 - the papilla fills up the entire proximal space; score 4 - the papilla is hyperplastic and excess tissue is present.

- PT-CP: distance (in $\mathrm{mm}$ ) between the papilla tip (PT) and the contact point $(\mathrm{CP})$

- PD and clinical attachment level (CAL) (in mm), bleeding on probing (BoP), and plaque (both in \%): measurements were taken at the mesial and distal aspects of the deficient papilla, both from the buccal and palatal side (i.e. four sites).

- Area of the "black triangle" (in $\mathrm{mm}^{2}$ )

- Esthetic appearance: the patient and the examiner evaluated the esthetic appearance of the region of the deficient papilla including that of the neighboring tooth and implant-supported crown by means of a VAS ranging from 0 to $100(0=$ no defect/best imaginable appearance, $100=$ worst imaginable defect/appearance).

The following parameter was judged after three months:

- Mucosal volume gain apically to the deficient papilla

The following parameter was assessed at baseline and after six months:

- Radiographic alveolar bone level at the implant next to the injection site $(\mathrm{mm})$ : Sample size calculation

\section{RESULTS}

Twenty two patients (11 per group) were allocated to either the test or control group. One patient of the control group had to be excluded shortly after the first injection, due to fracture and renewal of the adjacent implant-supported crown. Finally, 21 participants (12 female, 9 male; mean age $30 \pm 6.4$ years) received the allocated treatment and completed the follow-up period of six months. Thirteen papillae were between the central and lateral incisor, seven between the lateral incisor and the canine, and one between the canine and the first premolar; 14 implants were at position 12/22, four at position 13/23, and three at position 11 .

Injection of test and control substance did not cause any noticeable increase in papilla volume immediately after injection. Also, injection of test and control substance caused similar level of discomfort values, at both time points (VAS: $50-60$, on average). During the first week after injection, almost no discomfort was reported in the control group (mean VAS $\leq 5$ ), while low-to-moderate discomfort remained in the test group (mean VAS < 30). The difference between test and control groups in discomfort during the first week post-injection was statistically significant $(p<0.05)$ regarding the first and borderline significant regarding the second injection.

In the test group (HY group), adverse effects most likely triggered by the injection were reported in three patients. Two patients experienced severe pain and swelling of the lip after the second injection. In another patient, a painless granuloma of approximately six $\mathrm{mm}$ in diameter was observed above the mucogingival junction after the first injection; it persisted for >four weeks, but it was not detectable at the three-month check-up. No adverse effects were observed in the control group.

All included deficient papillae presented with a MPIS of 2 and none of them changed after treatment, that is, all remained at score 2. No significant differences in any of the clinical periodontal parameters (i.e., PD, CAL, plaque) were observed between the groups at any time point or within the groups over time. BoP was significantly higher in the control group compared with the test group at baseline and presented a significant increase in the test group from baseline to three months. No differences in the "black triangle" area were observed between groups at any observation time or within groups over time. Some minimal reduction of the "black triangle" could be detected only in a very few cases in the test group. Any recorded volume changes in the area of the attached gingiva adjacent to the deficient papilla after three months were minimal, and there were no significant differences between the test and control group. Finally, alveolar bone height at the implant site adjacent to the injection site remained basically stable for the six-month study period; 
no single case showed a $>0.5 \mathrm{~mm}$ difference between baseline and six months.

No significant differences in terms of aesthetic appearance between the test and control group at any time point or within the groups over time were observed by the patient or the examiner. A slight, but insignificant, improvement over time was reported by the patients in both groups. In general, the esthetic appearance of the deficient papilla was judged as acceptable by the patients and the examiner (VAS values on average $<30$ ) at all time points.

\section{CONCLUSIONS}

The researchers concluded that Injection of hyaluronan $(\mathrm{HY})$ adjacent to maxillary anterior implant-supported crowns did not result in any clinically conspicuous volume augmentation of deficient papillae.

\section{IMPLICATIONS FOR PRACTICE}

The benefits of $\mathrm{HY}$ experienced in other clinical applications have not been observed for the management of deficient papillae.

\section{Reference}

1. Bertl K, Gotfredsen K, Jensen SS, Bruckmann C, Stavropoulos A. Can hyaluronan injections augment deficient papillae at implant-supported crowns in the anterior maxilla? A randomized controlled clinical trial with 6 months follow-up. Clinical Oral Implants Research 2017; 28: 1054-61.

\section{Short (6-mm) dental implants versus sinus floor elevation and placement of longer $(\geq 10-\mathrm{mm})$ dental implants: a randomized controlled trial with a three-year follow-up.}

Bechara S, Kubilius R, Veronesi G, Pires JT, Shibli JA, Mangano FG. Clin. Oral Impl. Res. 28, 2017; 1097-1107.

Endosseous dental implants have become a predictable treatment option for applicable patients. ${ }^{1}$ The success rate of dental implants is associated with bone quality and quantity. Most implant failures occur in the maxillary molar region with poor bone quality. ${ }^{1}$ Other factors that may cause failure and difficulty in implant placement in the posterior maxilla are limited visibility, reduced interarch space and sinus pneumatization due to post-extraction bone resorption.

Short implants $(<10 \mathrm{~mm})$ were introduced recently as a new approach to simplify implant placement in compromised alveolar bone and to prevent possible damage to vital structures. The edentulous posterior maxilla is often characterized by reduced bone volume, due to severe post-extraction alveolar crest resorption coupled with age-linked sinus pneumatization. ${ }^{1}$ This anatomic limitation is a problem that can affect osseointegration and the fabrication of a functional and esthetic implant-supported restoration, dictating the need for reconstructive osseous surgery in order to restore a sufficient bone for implant insertion. Different bone augmentation techniques have been introduced to overcome this problem. Among these, maxillary sinus floor elevation has become the more reliable, commonly used procedure to increase bone height in the posterior maxilla. ${ }^{1}$ Although maxillary sinus floor elevation can be successfully employed to regenerate bone and allow the placement of implants of standard length recent studies have pointed out that this surgical intervention increases treatment duration, cost and is prone to complications such as graft failure and/or postoperative sinusitis. ${ }^{1}$

The placement of short $(<10-\mathrm{mm})$ dental implants represents a viable, minimally invasive alternative treatment solution for the prosthetic restoration of the

\author{
ACRONYMS \\ CBCT cone beam computed tomography \\ FPDs fixed partial dentures \\ ISQ Implant stability Quotient \\ MBL mean marginal bone loss \\ OPT orthopantomography \\ RFA resonance frequency analysis \\ SCs single crowns
}

posterior maxilla with limited amount of bone. The use of short implants requires no sinus floor elevation, reduces the risk of complications, treatment time and costs. Bechara and colleagues $(2017)^{1}$ reported on a threeyear follow-up randomized clinical trial that sought to evaluate whether short $(6-\mathrm{mm})$ dental implants placed in the posterior atrophic maxilla are a viable alternative to sinus floor elevation and placement of longer ( $\geq 10-\mathrm{mm})$ fixtures. The primary outcomes of the study were implant survival, stability, marginal bone loss, and complications associated with the two treatment options; secondary outcomes included treatment time and cost and patient satisfaction.

\section{MATERIALS AND METHODS}

The inclusion criteria for this trial were as follows:

1. (partial edentulism in the posterior atrophic maxilla;

2. post-extraction or healed sites (at least four months after extraction) with residual bone height $\geq 4 \mathrm{~mm}$ and width $\geq 5 \mathrm{~mm}$ under the maxillary sinus;

3. one to four adjacent implants required;

4. dentition in the posterior mandible for occlusal contacts;

5. age $\geq 18$; and

6. ability to sign an informed consent form. Patient eligibility in terms of bone dimensions was determined on orthopantomography (OPT) and cone beam computed tomography (CBCT) scans. 
Exclusion criteria were as follows:

1. severe systemic diseases that might contraindicate intervention;

2. uncontrolled diabetes mellitus;

3. immunocompromised status;

4. coagulation disorders;

5. radiotherapy;

6. chemotherapy;

7. alcohol or drug abuse;

8. pregnancy or lactation;

9. use of oral and/or intravenous amino-bisphosphonates;

10. untreated active periodontal infections;

11. active infection in the site of implant placement;

12. acute or chronic sinusitis;

13. history of sinus surgery; and bruxism.

14. Smoking, history of periodontal disease, and bruxism were recorded, but only the latter was an exclusion criterion.

In this prospective, randomized, controlled study, patients were randomly assigned either to receive one to four short (6-mm) implants (test group) or to undergo augmentation procedures and simultaneous placement of one to four standard-length ( $\geq 10-\mathrm{mm})$ implants (control group). Within the same group, patients could undergo bilateral treatment (i.e., short implants in the right and left posterior maxilla or bilateral maxillary sinus augmentation and placement of standard-length implants). Randomization was performed prior to surgery by opening a sequentially numbered sealed envelope corresponding to the patient recruitment number. In both groups of patients, dental implants featuring a tapered design with strong selfcutting threads (AnyRidge Implants) were used. These implants have an internal hexagon combined with a 5-mm-deep conical connection $\left(10^{\circ}\right)$, providing a tight seal and high mechanical strength, with built-in platform switching designed to maintain crestal bone and to increase soft tissue volume. The implants feature a novel nanostructured calcium-incorporated surface. In the control group, the augmentation procedures consisted of the insertion of collagenated porcine particulate bone graft (OsteoBiol GenOs) in a lateral window below the lifted membrane, with simultaneous implant placement. The test group (short implants) included both fresh postextraction sites and healed sites (defined as sites with at least four months of undisturbed healing after tooth extraction). In fresh post-extraction sockets, a flapless approach was used. The failing teeth were extracted as atraumatically as possible. In healed sites, a midcrestal incision was made, connected to two releasing incisions, and then a full-thickness flap was raised. In both fresh post-extraction sockets and healed sites, the surgeon placed short (6-mm) implants, being free to choose from the available implant diameters $(4-8 \mathrm{~mm})$ according to clinical indications and his preferences. All fixtures were placed at the bone crest level.

In the control group (standard-length implants in augmented sites), the maxillary sinuses were augmented using the lateral approach. After crestal incision and flap elevation, a lateral window was outlined and moved internally. After careful elevation of the Schneiderian membrane, the sinus cavity was partially filled with a collagenated porcine particulate bone graft; then, one to four 10-, 11.5-, 13-, or 15-mm fixtures were then surgically inserted. The operator was free to choose appropriate implant diameters $(4-8 \mathrm{~mm})$ on the basis of clinical indications and his preferences. Finally, the sinus cavity was completely packed and overfilled with bone graft particles, and the lateral window was covered with a pericardium porcine resorbable collagen membrane (Osteobiol Evolution).

In both groups, all implants were placed using a manual ratchet and then exposed to evaluate primary stability with a resonance frequency analysis (RFA) instrument. Implant stability quotient values were measured from the four sites (mesial, distal, buccal, and lingual sites) for each implant. An average value was calculated from the four measurements, rounded to a whole number, and regarded as the final Implant Stability Quotient (ISQ) value of the implant. A submerged healing protocol was selected for implants with an ISQ $<60$, whereas a nonsubmerged healing protocol (with placement of healing abutments) was selected in implants with an ISQ $\geq 60$. The mucoperiosteal flaps were repositioned and sutured over the cover screws (submerged healing) or around the healing abutments (non-submerged healing) using resorbable sutures.

After surgical procedures, all patients were prescribed oral antibiotics, $500 \mathrm{mg}$ amoxicillin plus clavulanic acid (Augmentin), every $8 \mathrm{~h}$ (three times per day) for six days. Postoperative pain was controlled with $600 \mathrm{mg}$ ibuprofen every $12 \mathrm{~h}$ for two days. Detailed instructions on oral hygiene were provided; chlorhexidine $0.2 \%$ mouth rinse (OralB) twice a day and a soft diet were recommended for two weeks. Patients were recalled and checked three days, 10 days (suture removal), and one month postoperation; they were not allowed to wear removable dentures up to one month postoperatively.

All implants were left unloaded to allow osseointegration over a period of four months; then, where necessary, the implants were uncovered and the cover screws were replaced with healing abutments. Impressions were taken, and provisional acrylic resin restorations, consisting of cemented or screw-retained single crowns (SCs) and fixed partial dentures (FPDs), were provided. The provisional remained in situ for four months, after which definitive restorations were provided. The definitive restorations comprised single crowns (SCs) and fixed partial dentures (FPDs). All definitive restorations were ceramometallic, screwed or cemented with temporary zinc oxide-eugenol cement. An accurate control of occlusion was performed, and protrusion and laterotrusion were evaluated on the articulator and intra-orally. Maintenance care was given every six months. All patients were included in an annual recall program.

At each annual inspection, an experienced, calibrated, independent examiner performed a careful clinical examination of the fixtures, peri-implant tissues, and prostheses. The primary outcome measures were implant survival, stability, marginal bone loss, and complications. All implant losses were considered as failures. Implant mobility in the absence of signs of infection, persistent/ recurrent infections (with pain, suppuration, bone loss), progressive marginal bone loss caused by mechanical overload, and implant fracture were indications for implant failure. Implant losses were divided into "early" (before the connection of the prosthetic abutment) and "late" (after the connection of the prosthetic abutment) failures. 
Resonance frequency analysis was employed to measure implant stability with a dedicated instrument. For each implant, ISQ values were measured from the four sites (mesial, distal, buccal, and palatal sites). The mean of all measurements was rounded to a whole number and regarded as the final ISQ of the implant. Afterward, the abutments were repositioned and installed on the implants, so that the prostheses could be re-inserted. ISQ values were obtained after implant insertion, at the delivery of the final restoration, and at the 1- and 3-year follow-up examinations.

Orthopantomographies were taken of each patient at baseline (immediately after implant insertion), delivery of the final restoration, and one and three years after implant placement. Radiographs were digitized, and saved in a dedicated folder. Peri-implant marginal bone levels were then measured using dedicated software. Marginal bone levels were measured on the mesial and distal sides of each implant and were compared at baseline, one and three year follow-ups.

Complications were biological and/or prosthetic. Biological complications included intraoperative complications (intraoperative bleeding), immediate postoperative complications (pain and swelling after surgery, acute sinus infection), late postoperative complications (chronic sinus infection, partial or complete graft failure), and problems in the function caused by an infectious process affecting the peri-implant tissues (peri-implant mucositis or peri-implantitis). The threshold to define peri-implantitis was set at a probing pocket depth $\geq 6 \mathrm{~mm}$ with bleeding on probing/suppuration and peri-implant bone loss $>3.0$ $\mathrm{mm}$. Prosthetic complications included failures or complications of prefabricated implant components (abutment loosening and abutment fracture) and superstructure-related failures (such as fracture of the metallic framework of the restoration, decementation/loss of retention, and ceramic fracture/chipping).

The evaluation of complications included identification of any complications that had affected the restorations over the three-year follow-up period. The secondary outcomes were treatment time and cost and patient satisfaction.

Patient satisfaction with both treatments (short implants and sinus floor elevation with placement of longer implants) was assessed. All patients were asked to give their perception of the received therapy by completing a questionnaire that dealt with function, esthetics, cleaning of the implantsupported restorations, satisfaction, and cost.

\section{RESULTS}

Fifty-three patients (19 males and 34 females) aged between 21 and 76 years (mean age: $48.1 \pm 15.1$ years, median: 48, 95\% Cl: 44.0-52.1) were included in this study. Among these, 15 (28.3\%) were smokers. According to the study design, the patients were randomly divided into two groups: 33 patients (10 males and 23 females) aged between 21 and 76 years (mean age: $47.5 \pm 16.2$ years, median: $48,95 \% \mathrm{Cl}$ : 42.0-53.0) were assigned to the test group (short implants without sinus floor elevation), and 20 patients ( 9 males, 11 females) aged between 28 and 75 years (mean age: $49.2 \pm 13.4$, median: 47.5 , 95\% Cl: 43.0-55.0) were assigned to the control group (sinus floor elevation with standard-length implants). Baseline demographics (gender, age, smoking habits, history of periodontal disease) did not reveal significant differences between the two groups. Twenty-one of the 53 enrolled patients had multiple indications for implant treatment (13 patients received two implants, two patients received three implants, four patients received four implants, and two received fiveimplants), so a total of 90 implants were installed. Forty-five implants were finally inserted in each group of patients.

In the test group, 33 patients received 45 implants whilst in the control group, 20 patients received 45 implants. In the test group, 36 implants were inserted in healed sites (at least four months after teeth extraction) of 25 patients, whereas nine implants were inserted in the extraction sockets (immediate implant placement) of eight patients. Among the test implants placed in healed sites, 24 (15 patients) were placed in ridges with a residual height of fourmm, whereas 12 (10 patients) were placed in healed ridges with a residual height of five to six $\mathrm{mm}$; among the control implants, 25 (10 patients) were placed in healed ridges with a residual height of four $\mathrm{mm}$, whereas 20 (10 patients) were placed in healed ridges with a residual height of five to six mm. In the test group, the most frequent indication was the restoration of single tooth gaps (24 implants were placed to support SCs); the least frequent indication was the restoration of partially edentulous patients with FPDs (21 implants were placed to support 11 FPDs). Conversely, in the control group, 21 implants were placed to support SCs, and 24 implants were inserted to restore partially edentulous patients (12 FPDs). There was a significant difference in the distribution of the implants between the two groups with respect to implant diameter (implant diameter was narrower in the control group than in the test group, $P<0.0001$ ); there were no significant differences in the distribution of implants between the two groups with respect to implant position and type of supported restoration.

Over the three year period after surgery, only two implant failures occurred, both in the same patient (control group). These failures occurred two months after surgery (before connection of the prosthetic abutment) and were classified as "early failures": they were caused by chronic sinus infection with loss of integration/implant stability. No further implant failures were observed in the control group after the delivery of prosthetic restorations. No implant failures occurred in the test group. Overall, oneyear survival rates of $97.8 \%$ (implant-based) and $98.2 \%$ (patient-based) were found. In the test group, the implant survival rate was $100 \%$ at the implant (45/45) and patient (33/33) levels; in the control group, the survival rates were 95.6\% (43/45) at the implant level and $95.0 \%(19 / 20)$ at the patient level. There were no significant differences in the one-year implant survival rate between the groups at both the implant level $(P=0.49)$ and patient level $(P=$ 0.38). One 76-year-old male patient in the test group (one implant) died two and a half years after implant placement and was consequently lost to follow-up: this patient was considered a dropout and was excluded from the study. No other patients dropped out of this study, so 52 patients were available for the three-year follow-up examination. Overall, three year survival rates of $97.8 \%$ (implant-based) and $98.1 \%$ (patient-based) were reported. The test group had a $100 \%$ implant survival rate at both the implant level 
(44/44) and patient level (32/32); the control group had a $95.6 \%$ survival rate $(43 / 45)$ at the implant level and a $95.0 \%$ survival rate $(19 / 20)$ at the patient level. There were no significant differences between the two groups at the three-year follow-up at both the implant level $(P=0.49)$ and patient level $(P=0.38)$

The mean ISQ values of the test and control groups did not differ at placement (test 68.2 vs. control $67.8, P=0.1$ ), at delivery of the final restoration (test 69.5 vs. control $69.4, P=0.9$ ), and after 1 year (test 71.0 vs. control 71.5 , $P=0.1$; however, at three years, the control group had a significantly higher mean ISQ than the test group (72.4 vs. 71.6, $\mathrm{P}=0.004)$.

Overall, a mean marginal bone loss (MBL) values of 0.18 $\pm 0.09 \mathrm{~mm}$ (median: 0.15 , range: $0-0.41$ ) and $0.24 \pm 0.11$ $\mathrm{mm}$ (median: 0.23, range: 0-0.5) were found at the one and three-year follow-up evaluations, respectively. Minimal bone changes around implants were observed with time; however, this difference was significant $(P<0.0001)$. Mean MBL was significantly higher in the control group than in the test group, both at one year $(0.14 \mathrm{~mm}$ vs. 0.21 $\mathrm{mm}, P=0.006)$ and at three years $(0.20 \mathrm{~mm}$ vs. $0.27 \mathrm{~mm}$, $P=0.01)$. With respect to the short implant subtypes, the MBL of implants placed in the post-extraction socket was significantly lower than that of control implants, both at one year $(P=0.03)$ and at trhree years $(P=0.02)$. $\mathrm{MBL}$ of implants placed in healed ridges with $3-4 \mathrm{~mm}$ of residual bone was significantly lower than that of control implants, both at one year $(P=0.003)$ and at three years $(P=0.005)$; however, in the five- to six-mm subtype, mean MBL did not differ from the values for the control group at one year $(P=0.5)$ and three years $(P=0.6)$. Finally, with respect to the short implants, there were no statistically significant differences in MBL between the different subtypes at one year $(P=0.2)$ and three years $(P=0.12)$.

No complications were reported for the test group. In the control group, 19 biological complications occurred: three were intraoperative (intraoperative bleeding) and 16 were immediately postoperative (one patient experienced pain and swelling after surgery, and 14 patients experienced swelling alone); finally, one patient experienced a late postoperative complication (chronic sinus infection with complete graft loss) which led to the loss of two implants. Significant differences were reported between the two groups with respect to intraoperative bleeding $(P=0.049)$ and swelling after surgery $(P<0.0001)$. Periimplant mucositis or peri-implantitis was not reported. All implant-supported restorations were free from prosthetic complications; no mechanical or technical complications were reported over the three-year period.

In the test group (short implants), the mean time needed for placement of one single implant was $19.1 \pm 7.1 \mathrm{~min}$ (median: 15, 25th-75th percentile: 15-20 min), whereas in the control group (sinus floor elevation with longer implants), the mean time needed was $32.2 \pm 8.5$ min (median: 30; 25th-75th percentile: 25-35 min). The sinus floor elevation procedure almost doubled the time needed for the intervention. The difference between the two groups was statistically significant $(P<0.0001)$.

The cost of both treatment modalities was calculated for one single implant limited to the surgery (without prosthetic treatment). The cost for the placement of one single implant in the short implant group was 700 euros (EUR), whereas in the longer implant group, the mean cost was $1322 \pm 490$ EUR. The sinus floor elevation procedure almost doubled the price for the intervention. The difference between the two groups was statistically significant $(P<0.0001)$.

There were no significant differences between the two groups with respect to satisfaction with function $(P=0.7)$, aesthetic outcome $(P=0.5)$, cleaning the implant-supported restorations $(P=0.6)$ and overall satisfaction with the treatment $(P=0.7)$. Perception of the cost of the therapy significantly favoured the test group $(P=0.03)$.

\section{CONCLUSIONS}

In the present randomized clinical trial, both short (6-mm) dental implants and longer $(\geq 10-\mathrm{mm})$ dental implants in combination with sinus floor elevation provided good results up to three years after loading; however, with 6-mm short implants, the treatment was faster and less expensive. Further, long-term randomized controlled trials on larger samples of patients are needed to confirm these results.

\section{IMPLICATIONS FOR PRACTICE}

The results of this trial suggest a significant benefit for patients having the short implants in terms of length of treatment and cost. Patient satisfaction and implant survival was high with both groups.

\section{Reference}

1. Bechara S, Kubilius R, Veronesi G, Pires JT, Shibli JA, Mangano FG. Short (6-mm) dental implants versus sinus floor elevation and placement of longer ( $\geq 10-\mathrm{mm})$ dental implants: a randomized controlled trial with a 3-year follow-up. Clin. Oral Impl. Res. 28, 2017; 1097-1107. 